\title{
Spatio-temporal dynamics of microlasers with chaotic ray dynamics
}

\author{
Stefan Bittner ${ }^{1,2}$, Stefano Guazzotti ${ }^{3}$, Yongquan Zeng ${ }^{4}$, Kyungduk Kim $^{1}$, \\ Qi Jie Wang ${ }^{4}$, Ortwin Hess ${ }^{3}$, Hui Cao \\ 1. Department of Applied Physics, Yale University, 06511 New Haven, CT, USA \\ 2. Chaire Photonique, LMOPS, CentraleSupélec, University Paris-Saclay, F-57070 Metz, France \\ 3. Department of Physics, Imperial College, London SW7 2AZ, United Kingdom \\ 4. School of Electrical and Electronic Engineering, Nanyang Technological University, 639798 Singapore
}

Broad-area semiconductor lasers used for high-power applications such as material processing and pump sources feature significant spatio-temporal instabilities [1]. Most attempts to stabilize them aim at reducing the number of transverse lasing modes via injection or feedback but fail at high powers. We demonstrate a different approach for stabilization via complex interference in asymmetric cavities with chaotic ray dynamics. This approach maintains multi-mode operation and is hence compatible with high-power operation [2].

We fabricated edge-emitting GaAs quantum well lasers by photolithography and dry etching. The dynamics of conventional stripe-shaped Fabry-Perot cavities [see Fig. 1(a)] was compared to those of asymmetric cavities featuring chaotic ray dynamics such as the cut-off circle [also called D-cavity, see Fig. 1(c)]. The properties of the passive modes of microcavities with chaotic ray dynamics have been intensively studied in the context of wave-dynamical chaos [3], however, little is known about their lasing dynamics. We measured the spatiotemporal dynamics of the microlasers using a streak camera with picosecond resolution.

The spatio-temporal dynamics of the emission from a Fabry-Perot cavity is shown in Figure 1(b). The emission intensity fluctuates on a sub-nanosecond time scale and is concentrated spatially in several filaments. The spatio-temporal instabilities result from the carrier-density dependence of the refractive index: a strong electric field depletes the carrier density, thus raising the refractive index locally and generating a self-focusing instability. In contrast, the spatio-temporal dynamics of a D-cavity, shown in Fig. 1(d), exhibits much slower and weaker fluctuations with a time scale of $50 \mathrm{~ns}$ or longer.

The differences in the dynamics are due to the structure of the lasing mode field distributions. Since the wave fronts in Fabry-Perot cavities travel almost parallel to the cavity axis, the transverse wavelength is of the order of several micron. An intensity variation on this length scale creates a lensing effect and thus a self-focusing instability. In contrast, the modes of a wave-chaotic D-cavity feature a fine-grained and pseudo-random spatial profile due to complex wave interference and have no dominant propagation direction. Thus, no lensing effect can be created, and self-focusing instabilities are prevented, resulting in a stable dynamics.

Furthermore, simulations of one-dimensional semiconductor lasers showed that the chaotic dynamics of a homogeneous cavity can also be stabilized by adding random variations of the refractive index profile to induce complex interference [2]. This demonstrates that the principle of modifying the interaction of lasing modes and active medium by means of a complex cavity environment is of general validity, and we anticipate applications in lasers as well as in other nonlinear wave-dynamical systems.
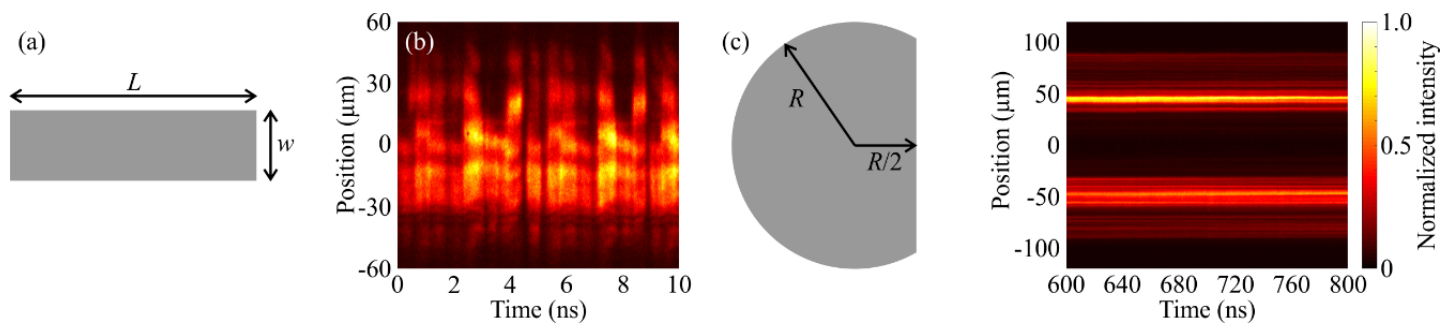

Fig. 1 Different cavity types and spatio-temporal images of their emission intensity. (a) Sketch of a Fabry-Perot cavity with length $L$ and width $w$ (not to scale) and (b) measured intensity distribution at the output facet of a Fabry-Perot laser with $w=60 \mu \mathrm{m}$, exhibiting very fast pulsations. (c) Schematic of a D-cavity with radius $R$ and (d) measured intensity distribution at the straight side wall of a D-cavity with $R=100 \mu \mathrm{m}$ at $0.6 \mu$ s after the start of the pulse, showing much slower and weaker variations.

\section{References}

[1] E. Gehrig and O. Hess, Spatio-Temporal Dynamics and Quantum Fluctuations in Semiconductor Lasers (Springer, Heidelberg, 2003). [2] S. Bittner, S. Guazzotti, Y. Zeng, X. Hu, H. Y1lmaz, K. Kim, S. S. Oh, Q. J. Wang, O. Hess, and H. Cao, "Suppressing spatiotemporal lasing instabilities with wave-chaotic microcavities," Science 361, 1225 (2018).

[3] H. Cao and J. Wiersig, "Dielectric microcavities: Model systems for wave chaos and non-Hermitian physics", Rev. Mod. Phys. 87, 61 (2015). 